\title{
Rule-based Visual Mappings - with a Case Study on Poetry Visualization
}

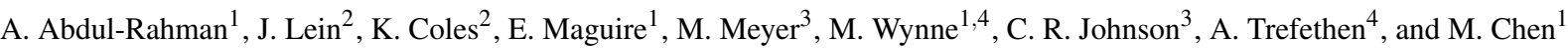 \\ ${ }^{1}$ Oxford e-Research Centre, and ${ }^{4}$ IT Services, University of Oxford \\ ${ }^{2}$ Department of English, and ${ }^{3}$ Scientific Computing and Imaging Institute, University of Utah
}

\begin{abstract}
In this paper, we present a user-centered design study on poetry visualization. We develop a rule-based solution to address the conflicting needs for maintaining the flexibility of visualizing a large set of poetic variables and for reducing the tedium and cognitive load in interacting with the visual mapping control panel. We adopt Munzner's nested design model to maintain high-level interactions with the end users in a closed loop. In addition, we examine three design options for alleviating the difficulty in visualizing poems latitudinally. We present several example uses of poetry visualization in scholarly research on poetry.
\end{abstract}

\section{Introduction}

A poem is a complex dynamic system. It features a variety of structural and relational information, including formal information (e.g., lines, stanzas), phonetic information (e.g., meter, intonation, timing), and semantic information (e.g., genres, words, repetition, sentiment). In addition, poems are studied from many different angles. Specific lenses or contexts for analysis might include the body of a poet's work, a historical period, a nation or geographic location, a group or movement, and so on. Although a poem is not a big data set, poets frequently devote hours to a close reading of a poem. In many ways, a close reading is a literary form of "data exploration", in which scholars pay close attention simultaneously to various individual linguistic, literary and sociological features, as enumerated above, as well as to the interplay and relationships among these features.

This paper is concerned with a user-centered design study on the visualization of poems. It could be said that one of the largest "professional gulfs" possible exists between computer scientists and poets. To a computer scientist, a poem appears to be an ordered sequence of letters - and may be considered as a multivariate data set. From this point of view, it would seem that one might define a set of variables within the complex information space of a poem and then employ a multivariate visualization technique such as parallel coordinate plots to discover various structures. Poets, on the other hand, insist that finding and reasoning through structural and relational information in a poem is at the heart of their close reading practice. This process is complicated, time consuming, and inherently experiential; the result is that interpretations vary from one person (or one context) to another. As one of the poets in this project explained, "Close reading involves moment-by-moment choices, where every choice activates some possibilities and deactivates others".

Such a "professional gulf" presented us with an interesting challenge and motivated us to demonstrate the potentially universal power of visualization by developing a suitable tool to help poets to explore the information spaces of poems. Following the nested design process [Mun09], the visualization researchers and the literary scholars formed a closed loop and engaged in a variety of collaborative activities, including joint workshops, brainstorming meetings, joint literature studies, close reading observations and recordings, questionnaires, requirements analyses, design evolution, and discussion of visualization results (Sections 3-6). In addition, one of the visualization researchers and one of the humanities scholars shared an office throughout the project.

We realized our technical solution would need to accommodate two potentially conflicting requirements: it would need to address a large number of variables (poetic attributes) while providing readers with the ability to make "moment-by-moment choices" as they explored visualizations. Inspired by the concept of the "artificial assistant" presented in [MHS07], we formulated a number of rules for reducing the options of visual mappings in an automatic and 
context-sensitive manner (Section 4). We designed and developed a rule-based interface for facilitating poem visualization (Section 5). We also developed and experimented with three different techniques for alleviating the difficulty in visualizing poems latitudinally (Section 6). This study showed that a rule-based approach shows promise in addressing the dual requirements mentioned above; it helped us make significant progress in developing visualization tools useful to close readers of poetry.

\section{Related Work}

In this section, we give a brief overview of the related work in visual literature analysis and other aspects of visualization, such as design process and visual encoding.

Visual Literature Analysis. There are many tools and techniques for exploring texts and documents. Some support the examination of the relationship between words using graphs (e.g., [Mil95, WV08, vHWV09, Pal]), while others use a radial, space-filling representation [CCP09]. A number of techniques (e.g., pixel-based visualization [KO07, $\mathrm{OBK}^{*} 08$ ], word cloud [VCPK09], and inkblots [AC07]) focus on viewing categorical information and statistical information about a document, revealing various signature patterns for comparative analysis. These techniques are designed primarily for distant reading [Mor05]. They are effective in many text analysis applications (e.g., [KO07, $\mathrm{DZG}^{*}$ 07], enabling examination of a large amount of text as a whole. For literary close reading, however, such approaches appear to offer limited scope and flexibility in supporting specific users' unique explorations. They neglect or ignore textual characteristics that poets and close readers value highly namely the complex and often subtle dynamic interplay among poetic features as they exist and develop in time and within particular contexts.

Our work addresses this gap by providing a visual and information-rich medium to literary scholars for exploring a large number of variables in a poem. While other programs [Pla06, CAT* 12 ] identify sonic structures and patterns to analyze, they do not capture or convey individual texts' dynamic complexity. The closest existing tool to ours is Myopia $\left[\mathrm{CGM}^{*} 12\right]$, a poetry visualization tool. The main difference between our work and Myopia is our support for visualizing many more poetic variables, such as dynamic temporality and relational data, and our use of rule-based mapping for visual encoding.

Other Aspects of Visualization. Our work builds on the research of others, including particularly relevant aspects of the following projects:

Mackinlay et al. [MHS07] presents a system that incorporates a default set of user interface commands for generating an effective graphical visualization. They also explore the issue of user experience in automatic presentation.
Munzner [Mun09] presents a four-level model for creating and evaluating a visualization system, where the output of each level is the input for the next one. Munzner examines a few visualization systems, such as visualization of genealogical graphs [MB05] and MatrixExplorer system [HF06], that utilize a nested approach in their development. Other frameworks proposed for designing and evaluating visualization systems include [AS04], [LPP* 06] and [VPF06]. Our tool adopts the nested design model [Mun09] as it allows us to engage on a high-level with the end users, while including their feedback in every level of the design process, thereby minimizing some of the drawbacks of evaluation.

There is a rich collection of work on visual encoding. For example, Bertin [Ber83] discusses visual encoding from a cartographer's point of view. Ware [War12] provides guidelines layout and design practices in data visualization and glyph design. Cleveland and McGill [CM84], and Heer and Bostock [HB10] explore the rankings of visual channels according to different types of data.

\section{Domain Problem Characterization}

In this section and the following three sections, we detail a user-centered design study by Munzner's [Mun09] nested model. The first level of this model is domain problem characterization.

Collaboration. This is a collaborative project between the University of Oxford and the University of Utah. The project team consists of computer scientists (specializing in visualization and related topics) and humanities scholars (specializing in poetry and linguistics). Our human-centered design process continues to involve literary scholars in various stages of the software development and implementation.

Initial Engagement. We arranged for visualization researchers to observe how literary scholars examine a small corpus by formulating and verifying hypotheses without the aid of visualization. In comparison with many other fields in the arts, humanities and social sciences, the discipline of poetry may typically involve smaller corpora and shorter text documents. During a Dagstuhl seminar, we established a list of typical scholarly questions that a literary scholar may ask during close reading. For example:

- What are the usages and temporal developments of different poetic elements, such as prosody, forms, syntax, etc.?

- Can visualization be used in analyzing at any given moment whether a transition or moment of innovation may be taking place in poetry as a whole and therefore how energized a current poetic moment may be?

- How do sound and other poetic features work in individual poems? Since all poems live in "time", even on the page, many new and interesting observations might be made with visualization for different poetic elements. 
These questions provided our first attempt to characterize the domain problem and user tasks. After a period of studying existing scholarship on text visualization, rhyme and meter, the full team met. In addition to project meetings, a special workshop was organized where many humanities scholars brought up a variety of interesting problems that might be subject to visualization solutions. The visualization researchers also attended a poetry reading and engaged in in-depth discussions with humanities scholars on sound and timing in poems, existing text visualization techniques, the relative merits of animation and static visualization, the use of colors in visualization, and so on. This first meeting, then, involved an intense period of mutual education.

Characterization. These few days of intensive collaborative activities helped the visualization researchers appreciate that poetic information is rich and highly compressed: that a word or even a syllable in a poem does not operate in a singular way. While poets are interested in statistical aggregation, their work is actually centered on the relationships between words, through which meaning, message, idea, impression, affect and impact arise and develop. Not content to settle on a single interpretation of a poem, they constantly suggest alternate interpretations of a specific component or an attribute, discussing and debating the relative "strength" or value of a given interpretation. Perhaps the most important observation made is that these interpretations seem much like hypotheses in scientific studies, except that there are usually many such hypotheses about just one poem. What became exciting was the idea that visualization could help literary scholars to make observations more effectively, to stimulate different interpretations, and to visually evaluate interpretations.

\section{Data and Operation Abstraction}

Data and Operations. In order to help visualization researchers gain a better understanding of the data, literary scholars in the project proposed a pilot study on the short poem "Night" by Louise Bogan [Bog95] with a specific emphasis on how sound develops in the poem through time. The initial, simple hypothesis to be evaluated was that the timing of reading this poem aloud might be sensitive to different accents. Two literary scholars from two different regions recorded their readings, and a recording of a computer reading was also added into the data set. These recordings were visualized with the words of the poem. Perhaps because there were not enough samples, we did not manage to discover sufficient evidence to support the hypothesis. The one thing we did discover is that the human interpretations were more similar to each other than they were to the computer interpretation, which appeared to weigh each syllable uniformly. In other words, the humans seemed to be interpreting the poem as they read it. Although this small exercise was inconclusive, the literary scholars were made aware of the importance and capability of multivariate visualization. This led to a focused team effort in which we worked to identify various poetic attributes that might be subject to computation and that might be visualized to stimulate hypotheses and evaluate them visually.

We began with a questionnaire about sound, designed to help us compile a list of observation tasks (i.e., operations) and the associated sonic attributes (i.e., variables) that one would wish to visualize for these tasks. We were able to capture 52 tasks, including, for example, the following:

- Which of the words in the poem rhyme with each other?

- How many of the vowels are unrounded vowels?

- How many groupings of repeated words are in the poem?

- Where are the caesuras in the poem?

- How many of the caesuras are masculine caesuras?

Abstraction. The team identified 33 measurable variables associated with 52 tasks. We then followed the first questionnaire with a second, which asked the poets to rate the importance of each variable from their point of view. The scholars eliminated 7 variables, because some are global statistics that do not vary within a single poem (e.g., line count), some are difficult to determine literally (e.g., semantic connection between two words), and some are less relevant to poems in English (e.g., pulmonic or non-pulmonic consonants). This leaves 26 variables, which are categorized primarily into two groups, namely the semantic group and the phonetic group. A table that details the 26 variables is given as part of the supplementary materials.

The variables in the semantic group are associated with the written words in Latin alphabet, while those in the phonetic group are associated with the sound and pronunciation of the words, for which we use the International Phonetic Alphabet (IPA) [Int99], a standardized representation of sounds for oral language. By using these two alphabets, we can compute most of the other variables - such as phonetic repetitions (e.g., end rhyme, internal rhyme, assonance, consonance, alliteration), word repetitions, and their frequency and connectivity - directly. In addition, we utilize external resources, such as sentiment ontology [Nie11], to attach semantic information to words, and UCREL CLAWS5 [UCR] to classify words grammatically. We employ a look up table to obtain articulatory features of vowels and constants [Pho].

We did explore the option of reducing the total number of poetic variables to a level that is more manageable in developing a visualization system. However, we found that removing some variables would significantly reduce the variety of observations that one could make in close reading. Our literary scholars were very reluctant to lose any variables. We were also aware that this list was not definitive or exhaustive, and would likely expand in the future. Thus, we decided to take on the challenge of handling 26 poetic variables in our goal to design a visualization system that literary scholars would find easy, inviting, and useful to engage as part of their close reading practice. 


\section{Encoding and Interaction Design}

For the purposes of visualization, a poem might be described as a sequence of words, usually divided into an arrangement of lines where each line may end with a rhyming sound depending on its form. In a poem, lines may also be grouped into stanzas. In the case of poems that are not divided into lines ("prose poems"), the decision not to use lines is an important one and must be taken into account in the design process. The ordering of words, lines (or lack thereof), and stanzas or "verse paragraphs" is thus an important attribute in the visualization, as it encodes temporal relationships between phonetic and semantic components. This attribute is thus encoded spatially as the basic contextual frame of a poem. Beyond the previously identified sonic requirements, therefore, we felt that it might be premature to introduce into our model those multivariate visualization techniques that do not explicitly encode spatial ordering of words, such as with parallel coordinate plots, multi-dimensional scaling and so on. We therefore focused on visual designs that depict poetic variables in conjunction with a display of the poem, either explicitly in textual form or implicitly in spatial location.

Let $V=\left\{v_{1}, v_{2}, \ldots, v_{n}\right\}$ be a set of all poetic variables, and $C=\left\{c_{1}, c_{2}, \ldots, c_{m}\right\}$ be a set of all visual channels available for visual mapping. One approach is to allow $v_{i}, i=$ $1,2, \ldots, n$ to map to any $c_{j}, j=1,2, \ldots, m$. Although encoding 26 poetic variables using 26 different visual channels in visualization is possible, in this scenario our target users would have to determine 26 different mappings through a naively designed control panel. Still, a control panel remains necessary as it is unlikely that literary scholars would want to visualize all poetic variables simultaneously. Moreover, the inclusion of all variables would induce clutter and interference between visual channels, impairing the user's experience of the visualization and ability to engage in visual exploration.

Alternatively, we could have a fixed mapping from $V$ to $C$. This would facilitate easy implementation. However, this would mean that some poetic variables would always map to more powerful visual channels [MRSS*12], while the visual analysis of other "less important" variables would be more difficult. This becomes problematic when some research tasks demand intensive observation of these "lesser" variables. Additionally, this approach would restrict the introduction of new poetic variables.

Inspired by the concept of the "artificial assistant" presented in [MHS07], we formulated four rules for reducing options for visual mappings in an automatic and contextsensitive manner. The goal is to provide a balanced solution to the visual and interaction designs. By intentionally avoiding fixed visual mapping, we can provide literary scholars with the freedom both to 1) choose different research foci, channeling their efforts towards specific regions in the hypothesis space, and 2) introduce new variables. Through a set of rules offering guidance on visual mapping, we can trans-

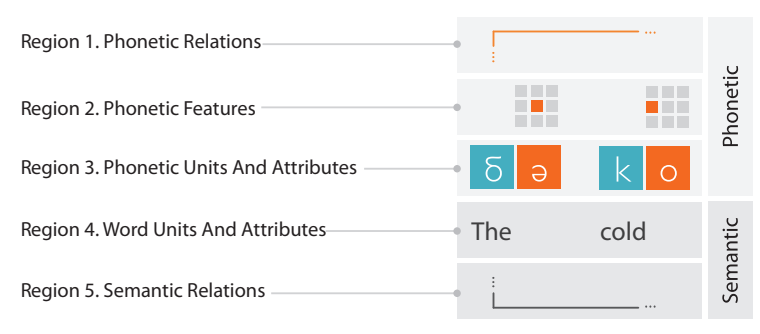

Figure 1: The division of the visual representation of a poem into 2 main domains (phonetic and semantic) and 5 regions.

fer some existing knowledge about best practices in visualization to literary scholars, while making interaction more scalable and less tedious.

Rule 1: Spatial Separation. Psychology research demonstrates that spatial memory and reasoning is an important factor that affect visual search (e.g., [Kos80, LS87, Kri00]). Meanwhile, spatial separation provides an effective means for alleviating the interference among visual channels and avoiding integrated visual channels [She64, MRSS*12]. Given numerous poetic variables, it is natural to group them based on some application-specific conceptual proximity, such as semantic meaning, task relevance, or a combination of several factors. One can then map such groups spatially to different regions, facilitating effective visual search and memorization. In our study, domain experts provided a grouping of the variables. This grouping resulted in our division of the visual representation of a poem into two main domains and five regions. As illustrated in Figure 1, the upper domain is designated for phonetic variables and consists of three regions, namely, 1) phonetic relations, 2) phonetic features and 3) phonetic units and attributes. The lower domain is designated for semantic variables, and it consists of two regions: 4) word units and attributes and 5) semantic relations.

The spatial separation based on conceptual grouping facilitates easy memorization of and visual search for a specific variable. It is not necessary for these five regions to be organized precisely as shown in Figure 1. In Section 6, we will discuss different layouts of the poem visualization. In this section, we focus the discussions on encoding and interaction design using the basic layout.

Regions 3 and 4 form the central axis of the basic spatial layout. To distinguish these two regions visually, region 3 has a boxed display for each phonetic unit, whereas region 4 has an appearance of free text. Each region can feature its own set of visual channels. For example, region 3 may offer the use of symbol, symbol color and/or intensity, symbol size, filled box color, intensity and/or texture, box outline color, style and thickness. Region 4 may offer symbol, symbol color and/or intensity, symbol size, background color and/or intensity, underline color, style, texture and/or thickness.

Regions 1 and 5 have similar data types and thus share 

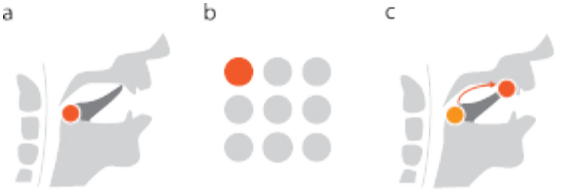

Figure 2: (a) A representation of the position of the tongue for a close front rounded vowel. (b) The glyph design in our system for a close rounded vowel. (c) A representation of the tongue moving from a close front to a close back. (d) The glyph shows the transition of the vowel positions where a light shade represents the previous position and a darker shade indicates the current position.

similar visual appearance. Nevertheless, the spatial separation helps minimize the confusion between information in these two regions. We use connection lines as the main visual objects in both regions. The visual channels available for these lines may include color, intensity, opacity, thickness, height, curvature, style and/or terminal shape. Region 2 is a glyph-based region for multivariate representations of phonetic variables. Currently, we provide glyphs designed for showing low-level features, such as characteristics and positions of each phonetic articulation in relation to the human vocal system.

Given a poetic variable $v$, we can use a look-up table to find its group categorization of $\Lambda_{\text {group }}(v)$. Similarly we can obtain the region categorization of $c$ as $\Theta_{\text {region }}(c)$. This allows us to define the first rule as:

$$
S_{R 1}(v, c)= \begin{cases}1 & \text { if } \Lambda_{\text {group }}(v) \text { is permitted in } \Theta_{\text {region }}(c) \\ 0 & \text { otherwise }\end{cases}
$$

The glyphs in region 2 are designed to supplement the phonetic symbols in region 3 as they help associate specific vowels or consonants to the "positions" and "geometries" of their sounds. We considered several different designs, starting from a mouth-shaped representation and finally settling on an abstract representation of $n_{\text {row }} \times n_{\text {col }}$ positions. Due to the size of the glyphs, we found that it was more difficult to recognize the positions in the designs that feature more realistic geometry.

The $3 \times 3$ positions of vowels encode (from left to right) front, central and back (which part of the tongue is raised); and (from top to bottom) close, mid and open (how far the tongue is raised). We use circles to represent a rounded vowel and squares to represent an unrounded vowel. To make the design more distinguishable, we also map the rounded and unrounded vowel positions to different colors orange and purple, respectively. Figure 2 shows an example glyph of a rounded vowel.

The $3 \times 2$ positions of consonants encode (from left to right) the two major classes of places of articulation: front articulation (labial, coronal), and back articulation (dorsal, radical, laryngeal); and (from top to bottom) the three major classes of manners of articulation: complete obstruent

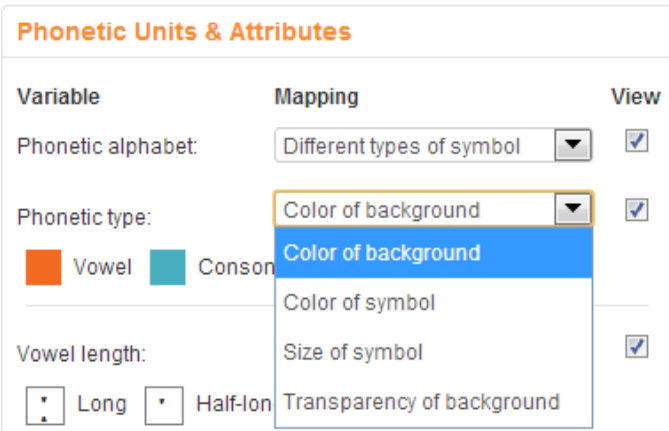

Figure 3: A snapshot of the final interface showing some of the variables with their visual mappings and legend.

(stop), partial obstruent (fricative, affricate), and sonorant. We use hollow and filled square in blue to represent voiceless and voiced sound respectively.

The colors used in the glyph region are consistent with the default color scheme in the phonetic unit region, where orange and blue represent the vowels and consonants. In addition, we use two different color intensities to encode the temporal transition of sound (Figure 2).

Rule 2: Type Compatibility. The poetic variables have four literal types: units (i.e., alphabets), attributes (i.e., nominal or ordinal), relations (i.e., sets or pointers), and attributes of relations (i.e., nominal or ordinal). Bertin [Ber83] proposes four criteria for determining the associative, selective, ordered and quantitative capabilities of a number of visual channels. However, Bertin does not provide a relational criterion that defines whether or not a visual channel is suitable for depicting the relation between 2 or more entities.

Based on these five criteria, we can define two vector functions $\Lambda_{\text {type }}(v)$ and $\Theta_{\text {type }}(c)$. $\Lambda_{\text {type }}(v)$ returns five $r e$ quirement scores, $\left[\lambda_{a}, \lambda_{s}, \lambda_{o}, \lambda_{q}, \lambda_{r}\right]$, indicating the requirements for $v$ to be associative, selective, ordered, quantitative, and relational respectively. Similarly, $\Theta_{\text {type }}(c)$ returns five capability scores, $\left[\theta_{a}, \theta_{s}, \theta_{o}, \theta_{q}, \theta_{r}\right]$. All these scores are within the range of $[0,1]$. With these two vector functions, we can define the rule for assessing type compatibility as:

$$
S_{R 2}(v, c)=\frac{\Lambda(v) \bullet \Theta(c)}{5\left(\lambda_{a}+\lambda_{s}+\lambda_{o}+\lambda_{q}+\lambda_{r}\right)}
$$

For example, the Latin alphabet type is a nominal variable, and we score its requirements as $[1,1,0,0,0]$. End rhyme relation is a relational variable, and we score its requirements as $[1,1,0,0,1]$. We score each visual channel according to its capacity, for instance assigning $[0.7,1,0.7,0,0]$ to the filled box color in region 3 , and $[0.3,1,0,0,1]$ to the connection line channel in region 1.

Rule 3: Channel Capacity. The capacity of a channel $c$ is the maximum number of distinguishable values it can represent. Such a maximum capability value depends not only on the representable range within the computer, such as the 

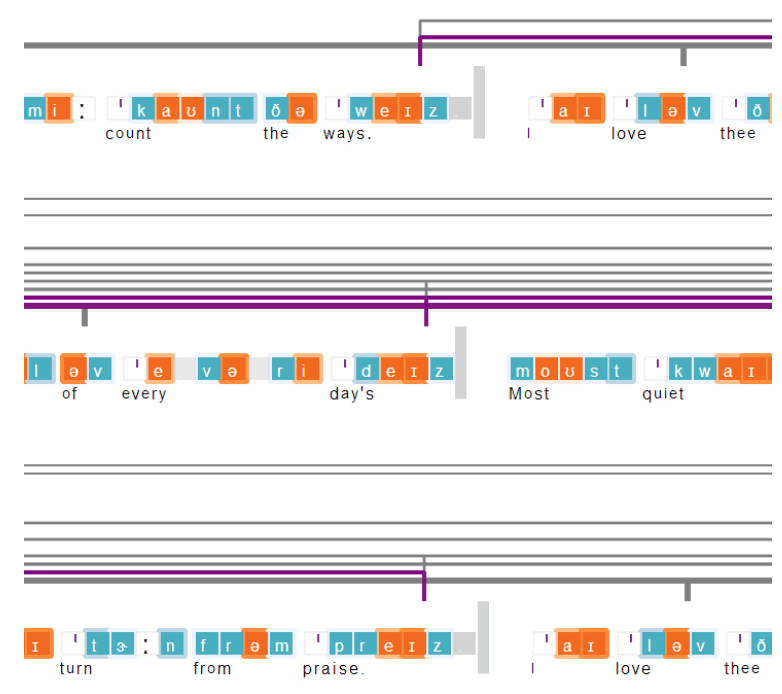

Figure 4: A sonnet showing its phonetic and rhyming connections, such as its internal rhymes, end rhymes and frequency of the IPA characters.

maximum length of line in pixels, or $256^{3}$ for colors. More importantly, it depends on many perceptual factors, such as just noticeable difference [BF73] and interference from nearby or integrated visual channels [She64].

For example, although the box outline in region 3 can be associated with visual channels such as color, thickness, and style, the capacity of the thickness channel is very limited as it has $\leq 3$ pixels of room for variation. Although the display may be capable of showing $256^{3}$ colors, the capacity of the color channel is in fact much lower, typically at about 512 colors [Hea96, War12], due to unreliable color perception and interference from the filled box colors. In general, it is desirable to use a visual channel of a higher capacity, though this is often in conflict with other requirements.

We assign each poetic variable with a minimal capacity requirement, $v_{\min }$ and a maximum requirement, $v_{\max }$. Meanwhile, each visual channel $c$ is given a capacity range, $c_{\text {lower }}$ and $c_{\text {upper }}$, where $c_{\text {lower }}$ indicates the ideal capacity limit, and $c_{\text {upper }}$ the strict maximum capacity. We define rule 3 for ranking the suitability of channel capacity as:

$$
S_{R 3}(v, c)= \begin{cases}1 & \text { if } v_{\max } \leq c_{\text {lower }} \\ 0 & \text { if } v_{\min } \geq c_{\text {upper }} \\ 0.5 & \text { otherwise }\end{cases}
$$

In general, the type compatibility rule $\left(S_{R 2}\right)$ attempts to determine how well a specific visual channel is suited for a given poetic variable. The channel capacity rule $\left(S_{R 3}\right)$ tries to ensure that there is enough "bandwidth" in the visual channel to display a desirable number of values for a poetic variable in a visually distinctive manner.

Rule 4: Already in Use. If a particular visual channel in a region is already assigned to a poetic variable, it is of course not appropriate to overload the same channel. Hence the 4th rule is defined as:

$$
S_{R 4}(v, c)= \begin{cases}0 & \text { if } \mathrm{c} \text { is in use } \\ 1 & \text { otherwise }\end{cases}
$$

Since each of $S_{R 1}, S_{R 2}, S_{R 3}, S_{R 4}$ is already normalized in the range of $[0,1]$, we can treat all of the rules as desirability functions [DS80]. The overall desirability $S$ for each pair of $v$ and $c$ is thereby:

$$
S(v, c)=\left(\prod_{i=1}^{4} S_{R i}(v, c)\right)^{1 / 4}
$$

We can define a threshold $0 \leq \tau<1$ such that when $S(v, c)>$ $\tau$, the visual channel $c$ is available to the poetic variable $v$ as an optional visual mapping. Figure 3 shows a pull-down menu with a significantly reduced number of visual mapping options. When users choose an option, a legend automatically appears, allowing users to inspect the suitability of particular visual mappings (e.g., type compatibility and channel capacity). The legends of the default mappings are given as part of the supplementary materials.

Evaluation. Evaluation processes were (and continue to be carried) out at different stages of the software design and implementation. Our team has conducted two main forms of evaluation so far: explicit evaluation, conducted through demonstration and trials of prototypes; and implicit evaluation, conducted through discussions about observable features and patterns depicted in visualizations. On several occasions, the evaluations led us back to the higher levels in the nested model. For example, after the evaluation of the rule-based visual mapping, the literary scholars organized a recorded close reading session so that visualization researchers could observe and appreciate the "levels of detail and complexity" that a poem visualization should ideally reach. Below, we give three examples of evaluations we conducted.

Figure 4 shows three sections of a sonnet. Each connection line represents a rhyme relation linking specific words to other rhyming words to reveal rhyming patterns across a poem. The height of the line is used to distinguish different rhymes, with internal rhymes shown in gray and end rhymes in purple. Thicker lines indicate those rhymes that occur more frequently. Such connection lines, have also been used to show musical refrains [Wat02] and allow for an easy overview of the distribution of rhyming patterns throughout the poem. During the demonstration and evaluation of this prototype, we discovered that the basic latitudinal layout does not support overview very well, though it works well in providing a detailed visualization. This led to brainstorming sessions on different layout algorithms.

Figure 5 shows a visualization for comparing the vowel positions of a free-verse poem, a sonnet and a nursery rhyme with those in a novel, a political manifesto and a paper in $\mathrm{Na}$ ture. The visualization researchers were initially puzzled by 


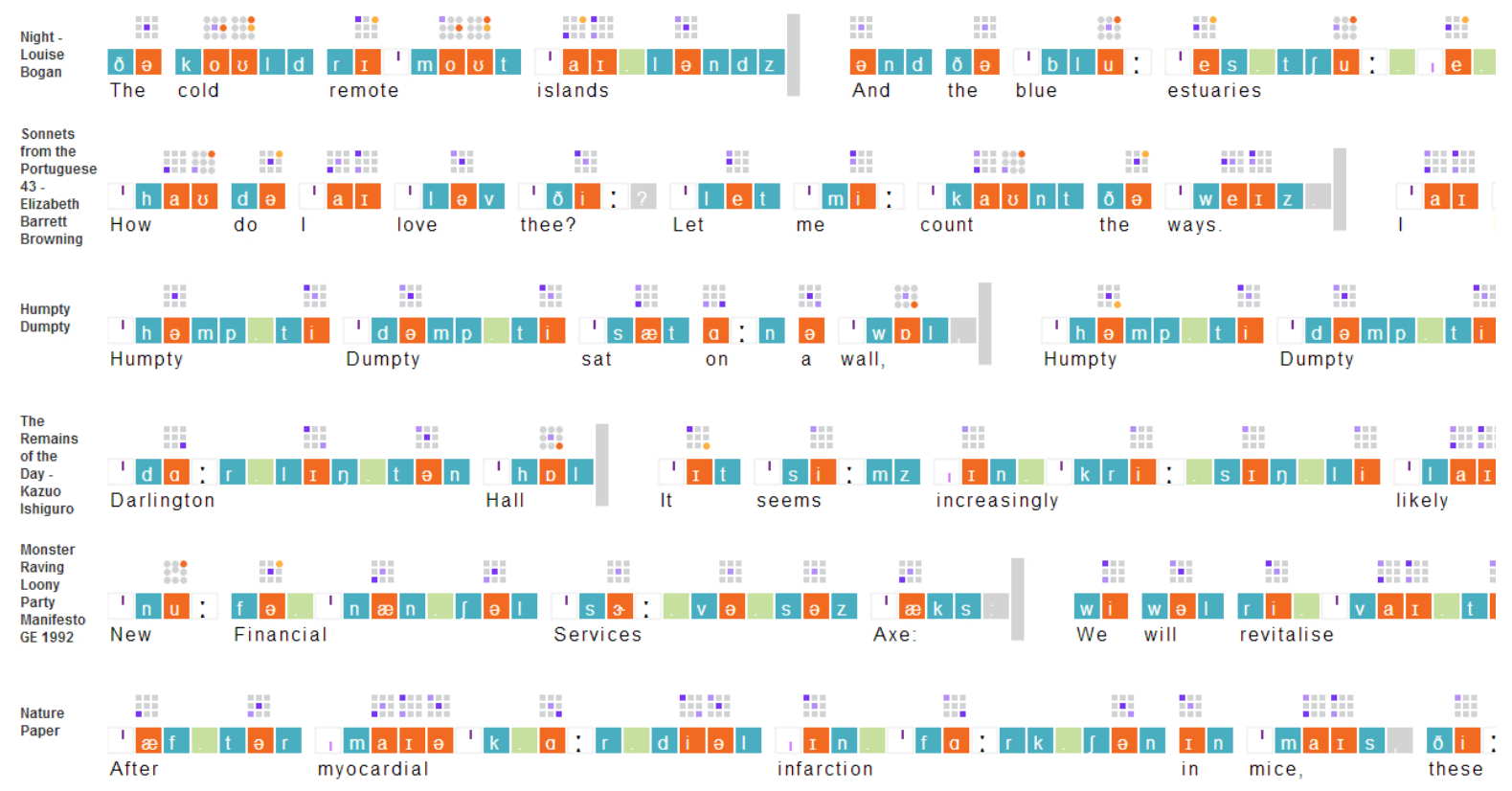

Figure 5: Visualization of poems and texts with a focus on vowel positions. The top three are poems: a free-verse poem, a sonnet and a nursery rhyme. The last three are prose texts: a novel [BNC07], a political manifesto [BNC07] and a Nature paper. The gray vertical bars indicate line breaks in poems or sentence breaks in other texts.

the visible difference in the glyph-based representations of phonetic features for poems and other texts. A Skype meeting was held to discuss this visualization result as an implicit evaluation. This visualization stimulated a few new hypotheses. For example, the two poems at the top clearly exhibit more dynamic changes in vowel positions than either of the prose pieces. This provides evidence to support the understanding of literary scholars that poets strive to enhance the density of their poems by creating dynamic sound through sonic recurrence and change in every word throughout the poem; it also leads to a hypothesis that language in prose texts or everyday use may have evolved for practical reasons to aid audience comprehension speed and accuracy by avoiding such sonic turbulence.

The above two examples of evaluation were carried out during the initial software prototyping stages. After the evaluation confirmed the merits of the rule-based visual mapping and interaction design, the desktop-based prototype was transformed to a web-based system called "Poem Viewer". The increased accessibility provided by this format enables further and more wide-spread ongoing evaluation. Thus far, the two poetry scholars have visualized 30 poems and poetic fragments. They used the tool mostly alone, but also once in the presence of a visualization scientist, who recorded their responses and - as is typical in ethnographic studies on visualization - asked what insight they had obtained. The poetry scholars responded that they would not likely look for insight from the tool itself. Rather, they would look for enhanced poetic engagement, facilitated by visualization. It is this enhanced engagement - not with the tool but with the poem - which might subsequently prompt new insights. At the same time, the poetry scholars observed that the most promising use of Poem Viewer was with the visualization of the sound placement in the mouth. They explained the merits of visualization: "While it takes at least as long to get information about mouth positions on a given word from the visualization as from saying the words and thinking about where they happen in the mouth, it is much faster to compare two different visualizations by eyeballing them for turbulence than to make such a comparison manually (or orally, if you will)."

As the second example (Figure 5) shows, if sound placement is used as the measure, poems are more musically various or disrupted than prose. Using the vowel positions, poetry scholars were able to observe sound developing across the texts and linking lines and stanzas through patterns of recurrence and change, which are of intense interest to poetry scholars. For example, they can see the repetition of the long "o" sound linking "cold" and "remote" evolving into the repeated long "u" sound of "blue" and "estuaries" in the free-verse poem. Such a transformation cannot be seen easily either in simple alphabet spellings or IPA character representations of syllables. Something that is normally heard and has to be related using a mental image can now be visualized with the help of glyphs. The scholar was also able to use the visualization to watch the evolution of the vowel from "I" to "love" to "thee" in the sonnet.

Still another interesting example of this visualization's 


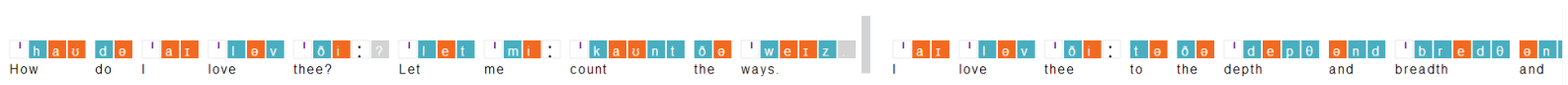

(a) Basic visualization of a sonnet (only $15 \%$ of the poem is being displayed).

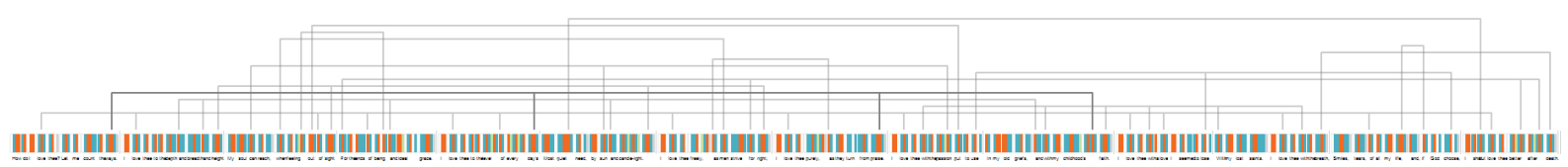

(b) A sonnet that has been downsampled with uniform scaling.

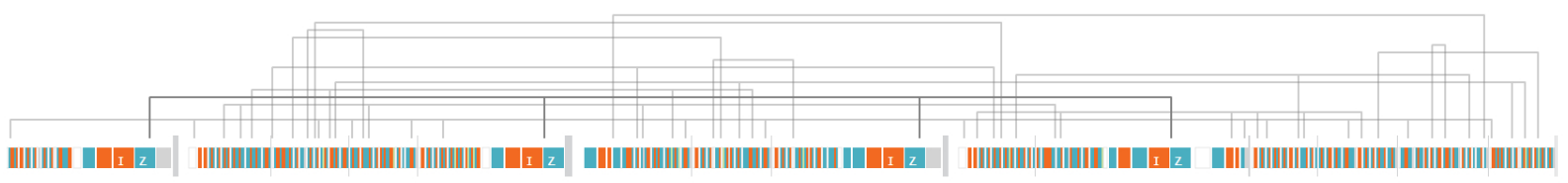

(c) Focus+context of a sonnet using a non-uniform scaling.

Figure 6: Three different latitudinal layouts of a poem.

usefulness occurs in the nursery rhyme, "Humpty Dumpty", in particular in looking at the phrase "sat on a wall". Using the IPA character, we can see that "on" and "wall" are repeating the same vowel sound even though they are not using the same alphabetic letters. Without the vowel position glyphs, poetry scholars may not consciously think about the fact that "sat on the wall" resides in the lower register, moving only briefly to the neutral mid-point. This is interesting since the positioning links those words even though they do not all rhyme. The link is further strengthened by the fact that all the words are single-syllable words. The combination of the lower register and the single syllable makes the phrase more emphatic than, for example, the phrase "Humpty Dumpty", which is both multi-syllabic and resides in the mid-to-upper vowel register. This is an observation that a poetry scholar may come to much more slowly - if at all - without the tool.

\section{Algorithm Design}

The major algorithmic development in this project is the layout of a poem visualization. On one hand, the basic latitudinal layout enables the phonetic and semantic connections across a poem. On the other hand, as identified in a formal evaluation session mentioned in the previous section, it does not support overview very well. Figure 6(a) shows a section of such a basic visualization of a poem. For each character/box that is to be displayed, we assign a set of display geometries such as $x, y$, width and height. For the basic visualization, the rendering moves horizontally by the width, $x_{\text {new }}=x_{\text {previous }}+$ width.

Figure 6(b) shows a basic visualization that has been downsampled in the $x$ direction with uniform scaling. The poem is deformed horizontally, where $x_{\text {new }}=x_{\text {previous }}+$ width $*(\eta)$. $\eta$ is a scaling factor $<1$. An advantage of using this approach is that the user is provided with an overall view of the poem with its connecting arcs. A disadvantage is that the user is not able to see any phonetic or Latin characters.

Poem Viewer demonstrates an alternative approach to the uniform downsampling method. As shown in Figure 6(c) when a particular rhyme is selected, the latitudinal visualization ensures that the corresponding regions along the $x$-axis are still in focus, while the rest of the visualization is downsampled to provide the context. Algorithmically, we achieve this by first establishing a set of focal points, $f x_{1}, f x_{2}, \ldots, f x_{k}$ according to a selected connection line or a rhyming pattern. We then create a curve with a Gaussian function at each focal point. We finally render the overview for using this curve as a transfer function for determining the rate of downsampling. Using this approach, the user not only has an overview of the rhyming connections across the poem but is also able to identify the connecting rhyming characters.

The conventional layout of a poem is two-dimensional. We thus investigated the feasibility of visualizing poems in their "organic" layout. The main difficulty is that connection lines running among other visual channels, such as symbols and filled boxes, causes extensive visual cluttering. Poem Viewer thus implements a layout that allows connecting lines to run on either the left- or right- hand sides of the poem, as shown in Figure 7. The semantic connections, such as repeated words, are displayed on the left hand side of the poem, where the height of the arc indicates the different groupings of repeated words, while the right hand side is used for displaying the phonetic connections in the poem. For internal rhyme relations and word-based semantic relations, the arc height alone is not sufficient to separate the different groups. We thus introduce an offset mechanism to relax the connection terminators in $y$ direction. For each line in a poem, we store its grouping of repeated words (or internal rhymes), moving from the most to least frequent, (top 


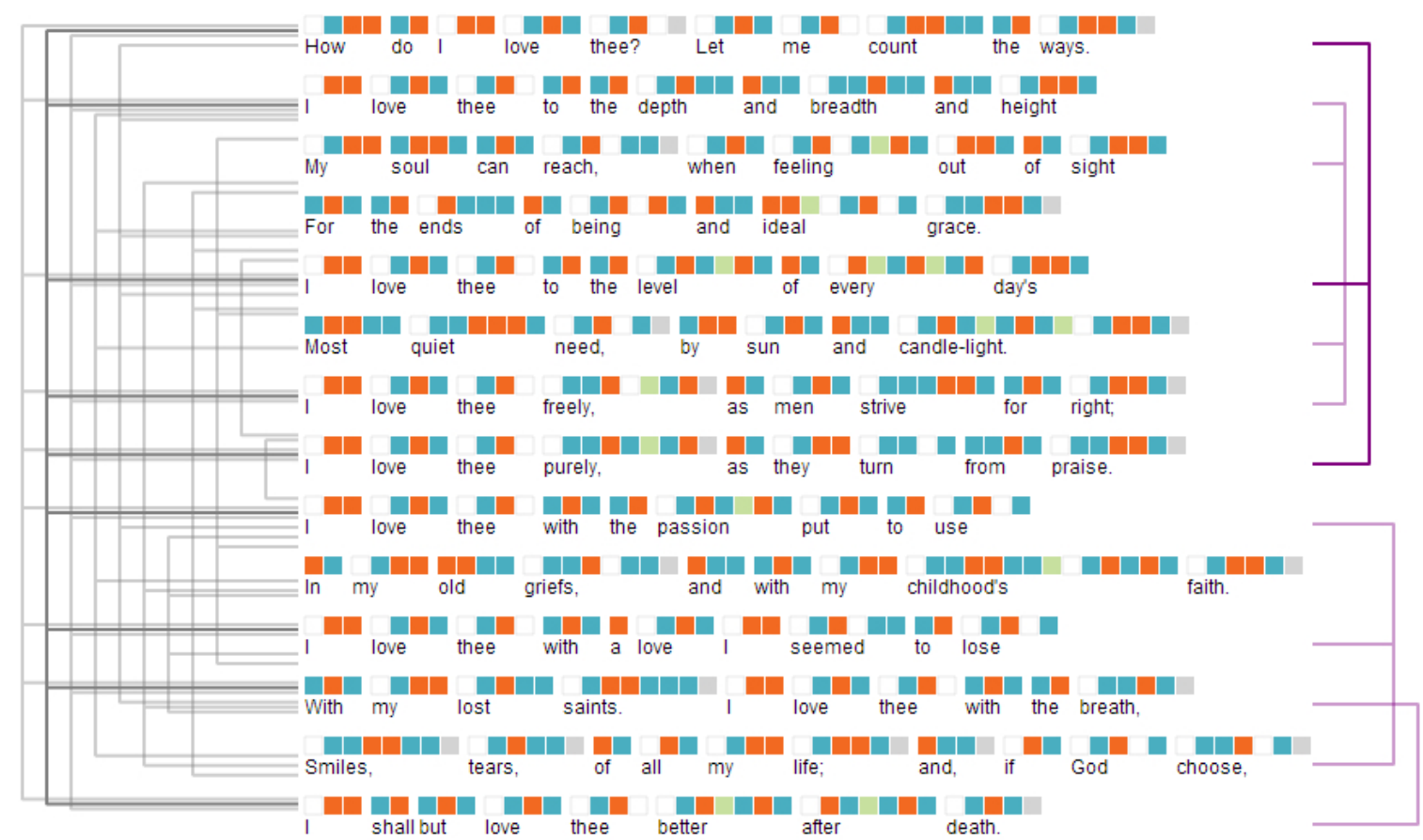

Figure 7: A structurally laid out poem with its phonetic and semantic connections.

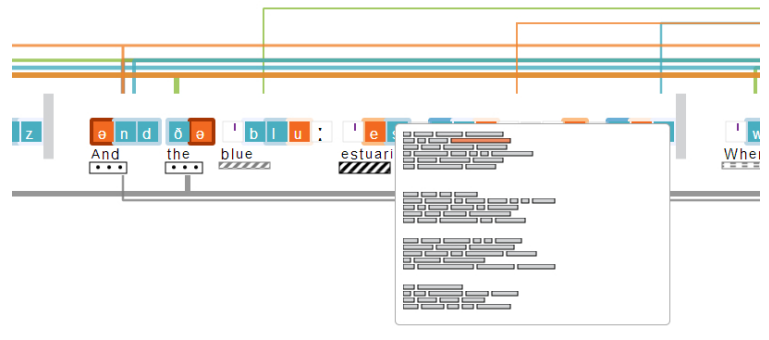

Figure 8: A user can interact with the visualization to view the location of a word in the structured layout. The texture under each word represents a grammatical "part of speech".

to bottom). As we proceed through the list, we offset its $y$ position by a constant $\delta$. The main disadvantage is that one cannot associate individual connection lines with any particular word or internal rhyme within a line of text.

We have observed that poetry scholars tend to know the poem under study extremely well. Hence, the basic latitudinal visualization shown in Figure 6(a) remains useful in close reading. We can augment the basic visualization with a context view interactively. As shown in Figure 8, the user can click on a word to see its position in the context of the whole poem. Other forms of interaction include selecting and highlighting a specific word or relation throughout the poem. All four layout algorithms can perform in real-time on an Intel laptop (2.8GHz, $8 \mathrm{~GB}$ of RAM). This makes the algorithms suitable for interactive visualization.

\section{Conclusions}

In this paper, we reported on our experience of a fascinating collaboration between computer scientists and literary scholars and on our design study on poetry visualization. We found that the nested model by Munzner [Mun09] offers an effective framework for organizing different types of user engagements. Through this intensive collaboration, we were able to formulate a rule-based solution to address the need for high-dimensional multivariate visualization of poems a notion that was difficult for both computer scientists and literary scholars to grasp at the beginning of our work. In addition, we developed a user interface to support the tasks of visual mapping along with several layout algorithms. We are in the process of transforming the prototype system into a browser-based tool for easy installation and use. We will also conduct a number of focused studies on some literary hypotheses generated during this project.

\section{Acknowledgments}

This work was funded jointly by NEH (USA) and JISC (UK) under the Digging Into Data Challenge program.

\section{References}

[AC07] ABbasi A., Chen H.: Categorization and analysis of text in computer mediated communication archives using visualization. In Proc. 7th ACM/IEEE-CS Joint Conf. Digital Libraries (2007), pp. 11-18. 2 
[AS04] AMAR R., STASKo J.: A knowledge task-based framework for design and evaluation of information visualizations. In Proc. IEEE Symp. Information Visualization (2004), pp. 143150. 2

[Ber83] BERTIN J.: Semiology of Graphics: Diagrams, Networks, Maps. University of Wisconsin Press, 1983. 2, 5

[BF73] Booth D., Freeman R. J.: Discriminative measurement of feature integration. Acta Psychologica (1973). 6

[BNC07] BNC: The British National Corpus, version 3 (BNC XML Edition). Distributed by Oxford University Computing Services on behalf of the BNC Consortium, 2007. URL: http: //www.natcorp.ox.ac.uk/. 7

[Bog95] Bogan L.: Night. The Blue Estuaries: Poems 19231968, 1995. 3

[CAT*12] Clement T., Auvil L., Tcheng D., Capitanu B., Monroe M., Goel A.: Sounding for Meaning: Analyzing Aural Patterns Across Large Digital Collections. In Digital Humanities (2012). 2

[CCP09] Collins C., Carpendale M. S. T., Penn G.: Docuburst: Visualizing document content using language structure. Computer Graphic Forum 28, 3 (2009), 1039-1046. 2

[CGM*12] Chaturvedi M., Gannod G., Mandell L., Armstrong H., Hodgson E.: Myopia: A Visualization Tool in Support of Close Reading. In Digital Humanities (2012). 2

[CM84] Cleveland W. S., MCGill R.: Graphical perception: Theory, experimentation, and application to the development of graphical methods. Journal of the American Statistical Association 79, 387 (1984), 531-554. 2

[DS80] DERRINGER G., SUICH R.: Simultaneous optimization of several response variables. Journal of Quality Technology 12, 4 (1980), $214-219.6$

[DZG*07] Don A., Zheleva E., Gregory M., TARKan S., Auvil L., Clement T., Shneiderman B., Plaisant C.: Discovering interesting usage patterns in text collections: Integrating text mining with visualization. In Proc. 16th ACM Conf. Info. and Knowledge Management (2007), pp. 213-222. 2

[HB10] HeER J., Bostock M.: Crowdsourcing graphical perception: Using mechanical turk to assess visualization design. In Proc. of the SIGCHI (New York, 2010), pp. 203-212. 2

[Hea96] Healey C. G.: Choosing effective colours for data visualization. In Proc. 7th Conf. Visualization (1996). 6

[HF06] Henry N., FeKete J.-D.: MatrixExplorer: A dualpresentation system to explore social networks. IEEE Trans. Visualization and Comp. Graphics 12, 5 (2006), 677-684. 2

[Int99] InTERnational Phonetic Association: Handbook of the International Phonetic Association. Cambridge University Press, 1999. 3

[KO07] KeIM D. A., OELKE D.: Literature fingerprinting: A new method for visual literary analysis. In IEEE VAST (2007), pp. 115-122. 2

[Kos80] Kosslyn S. M.: Image and Mind. Cambridge: Harvard University Press, 1980. 4

[Kri00] KRISTJÁNSSON A.: In search of remembrance: Evidence for memory in visual search. Psychological Science 11, 4 (2000), $328-332.4$

[LPP*06] Lee B., Plaisant C., Parr C. S., Fekete J.-D., HENRY N.: Task taxonomy for graph visualization. In Proc. AVI BELIV Workshop (2006), pp. 1-5. 2

[LS87] LARKIN J. H., Simon H. A.: Why a Diagram is (Sometimes) Worth Ten Thousand Words. In Cognitive Science (1987), vol. 11 , pp. $65-99.4$
[MB05] MCGUFFIN M., BALAKRISHNAN R.: Interactive visualization of genealogical graphs. In IEEE Symp. Information Visualization (2005), pp. $16-23.2$

[MHS07] Mackinlay J., Hanrahan P., Stolte C.: Show Me: Automatic presentation for visual analysis. IEEE Trans. Visualization \& Comp. Graphics 13, 6 (Nov. 2007), 1137-1144. 1, 2,4

[Mil95] Miller G. A.: WordNet: A lexical database for English Communications of the ACM 38, 11 (Nov. 1995), 39-41. 2

[Mor05] MoRETTI F.: Graphs, Maps, Trees: Abstract Models For A Literary History. Verso, 2005. 2

[MRSS*12] Maguire E., Rocca-Serra P., Sansone S.-A., DAvies J., Chen M.: Taxonomy-Based Glyph Design - with a Case Study on Visualizing Workflows of Biological Experiments. IEEE Trans. Visualization \& Comp. Graphics 18, 12 (2012), 2603 - 2612. 4

[Mun09] MUNZNER T.: A nested model for visualization design and validation. IEEE Trans. Visualization \& Comp. Graphics 15, 6 (Nov. 2009), 921-928. 1, 2, 9

[Nie11] NIELSEN F. A.: A new ANEW: Evaluation of a word list for sentiment analysis in microblogs. In Proc. ESWC Workshop on 'Making Sense of Microposts' (May 2011), pp. 93-98. 3

[OBK*08] Oelke D., BaK P., Keim D., Last M., Danon G.: Visual evaluation of text features for document summarization and analysis. In IEEE VAST (Oct. 2008), pp. 75 -82. 2

[Pal] PALEY W. B.: TextArc. URL: http: //www. textarc. org/. 2

[Pho] Photransedit: Text to phonetics. http://www.photransedit.com/Online/ Text2Phonetics.aspx. 3

[Pla06] Plamondon M. R.: Virtual verse analysis: Analysing patterns in poetry. Literary and Linguistic Computing 21, suppl 1 (2006), 127-141. 2

[She64] SHEPARD R.: Attention and the metric structure of the stimulus space. Journal of Mathematical Psychology 1, 1 (1964), $54-141.4,6$

[UCR] UCREL: Claws part-of-speech tagger for English. URL: http://ucrel.lancs.ac.uk/claws/. 3

[VCPK09] Vuillemot R., Clement T., Plaisant C., KuMAR A.: What's being said near "Martha"? Exploring name entities in literary text collections. In IEEE VAST (2009), IEEE, pp. 107-114. 2

[vHWV09] van Ham F., Wattenberg M., Viegas F. B. Mapping text with phrase nets. IEEE Trans. Visualization \& Comp. Graphics 15, 6 (Nov. 2009), 1169-1176. 2

[VPF06] Valiati E. R. A., Pimenta M. S., Freitas C. M. D. S.: A taxonomy of tasks for guiding the evaluation of multidimensional visualizations. In Proc. AVI BELIV Workshop (2006), pp. 1-6. 2

[War12] WARE C.: Information Visualization: Perception for Design, 3rd ed. Morgan Kaufmann Publishers Inc., San Francisco, CA, USA, 2012. 2, 6

[Wat02] WatTenberg M.: Arc diagrams: Visualizing structure in strings. In Proc. IEEE Symp. Information Visualization (2002), pp. $110-116.6$

[WV08] Wattenberg M., Viégas F. B.: The Word Tree, an interactive visual concordance. IEEE Trans. Visualization \& Comp. Graphics 14, 6 (Nov. 2008), 1221-1228. 2

(C) 2013 The Author(s) 